\title{
Demonstrating the Value of 3D Macromolecular Structures
}

\section{STEPHEN BURLEY. RCSB PROTEIN DATA BANK}

While the DNA serves as a symbol of science to the general public, mentions of proteins usually inspire thoughts of nutrition. Crystals can be either Swarovski-related or "new age". How can we demonstrate the value and impact of structural data in this cultural landscape?

RCSB PDB and PDB-101 offer various tools, from wwPDB Validation Reports to Molecule of the Month articles to videos, that help tell the stories behind PDB structures.

RCSB PDB is additionally exploring ways of demonstrating the impact of the data contained in the PDB archive. Website analytics, bibliometrics and economic studies provide information about how structural biology impacts everything from Agriculture to Zoology. 